\title{
REVIEW OF COMPACT COMMERCIAL ACCELERATOR PRODUCTS AND APPLICATIONS
}

\author{
Y. Jongen, Ion Beam Applications (IBA), Louvain-la-Neuve, Belgium
}

\begin{abstract}
Application-oriented accelerators are generally built commercially, and they often emphasize other qualities than the accelerators for research. These accelerators are generally specialized for a specific application, and are therefore very simple and inexpensive to operate. The author will review some products and applications in the field of medicine, such as the production of radio-isotopes and radiotherapy, as well as in the industrial field, such as sterilization applications and the modification of materials.
\end{abstract}

\section{INTRODUCTION}

Historically, particle accelerators were developed initially for nuclear, then for particle physics research. From this research resulted applications of accelerators in the field of medicine and industry. These application-oriented accelerators are generally built commercially, and they often emphasize other qualities than the accelerators for research. Research frequently requires energies or beam qualities at the limit of the existing technologies. Accelerators for research offer the largest flexibility in term of particles and beam properties, but are more complex, more expensive and often require large and highly qualified staff to operate and maintain them. In contrast, most applications are done with low to moderate energy protons or electrons, but often with large average beam power. The accelerators are generally specialized for a specific application, and are therefore very simple and inexpensive to operate. In the field of medicine, typical applications include the production of radioisotopes for medical diagnostic or the production of electrons, protons or fast neutron beams for cancer therapy. In the industrial field, high power electron beam are used for sterilization and for the modification of materials.

\section{PRODUCTION OF RADIOISOTOPES FOR IMAGING AND DIAGNOSIS IN NUCLEAR MEDICINE}

Radioisotopes are essential in diagnosis and study of the human functions. The access to the full range of radioisotopes used in nuclear medicine is vital to the early diagnostic and treatment of a wide variety of problems including cancer and heart disease. Various radioisotopes are produced [1] with cyclotrons, according to the organ that must be represented: Gallium 67, Iodine 123, Thallium 201, among others. To produce such radioisotopes, a medium energy cyclotron is a suitable device. In Europe, in the USA and in Japan, commercial companies operate several cyclotrons in the same facility. Many important hospitals and clinics are now also equipped with cyclotrons producing radioisotopes for their own use or for a distribution on a regional scale.

All this is possible because of the high degree of automation, reliability and simplicity characterising the cyclotrons used nowadays for radioisotope production. The evolution of the cyclotrons dedicated to this application is quite interesting [2]. The first cyclotrons produced commercially for the production of medical r.i. were "classical", positive ion, isochronous cyclotrons, similar to their counterparts built for nuclear physics research, where the beam was extracted using an electrostatic deflector. The heat dissipation limits in the deflector septum limited the extracted beam power to a couple of $\mathrm{kW}$, but higher intensities, typically several hundred $\mu \mathrm{A}$ were available on internal targets. The Cyclotron Corporation (TCC), based in Berkeley (Ca), produced between 1968 and 1984 in excess of 30 cyclotrons based on this technology. A major step was made in the early 80's when TCC proposed a $42 \mathrm{MeV}$ cyclotron accelerating negative ions, produced by an internal P.I.G. source, and extracting by stripping. Extracted beam currents exceeding $200 \mu \mathrm{A}$ up to $40 \mathrm{MeV}$ were available. Thanks to the stripping extraction, variable energy extracted beams could be obtained in a fixed field, fixed frequency cyclotron. The simultaneous extraction of two beams was also, in principle possible. However, the use of an internal ion source meant a poor vacuum in the cyclotron accelerating tank, and significant amounts of beam were lost in the median plane by stripping on the residual gas. The need to deal with these large beam losses resulted in a quite complex design of the dees and of the R.F. systems, and resulted, at least in the beginning, in a lower reliability. This cyclotron was, initially, a commercial success. Six CP42's were ordered and built, even before the prototype was fully tested. Delays in the commissioning of these cyclotrons contributed eventually to the bankruptcy of TCC. After this significant failure, negative ion technology was rejected by radioisotope producers for some time, in favor of more classical positive ion isochronous cyclotrons, like the MC40 model, made by the Scanditronix company in Upsala. 


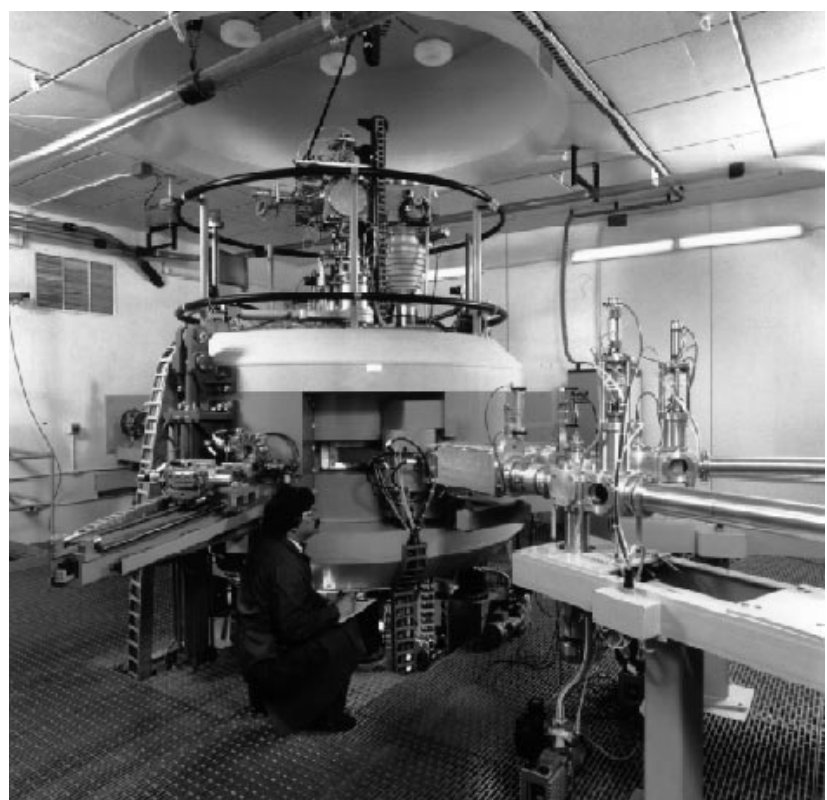

Fig. 1. A CYCLONE 30 cyclotron manufactured by IBA

The next major step in radioisotope production cyclotrons was the introduction, in 1987, by IBA, of the CYCLONE 30. It is a $30 \mathrm{MeV}$, fixed-field, fixed-frequency, $\mathrm{H}^{-}$ cyclotron with extraction by stripping, dual beam and variable energy. The first major improvement of this model was the use of an external, multicusp ion source for the production of the negative ions. The ions are injected axially into the cyclotron magnet, so that the neutral gas released by the ion source can be pumped externally. The second major improvement was the introduction of the "deep valley" magnet design, combining the advantage of solid pole and separated sectors cyclotrons, and allowing to improve significantly the power efficiency of the accelerator. Typically, previous positive ion cyclotrons were using $300 \mathrm{~kW}$ of electricity to get $3 \mathrm{~kW}$ of extracted beam (1\% power conversion efficiency), while the CYCLONE 30 required only $90 \mathrm{~kW}$ of electricity to produce $15 \mathrm{~kW}$ of extracted beam (16\% power conversion efficiency). The CYCLONE 30 was the first cyclotron to demonstrate the possibility to have a beam loading exceeding $50 \%$ of the total R.F. power. Today, the CYCLONE 30 is the world standard for radioisotope production. A total of 17 CYCLONE 30 have been sold up to now by IBA. Since a number of years, another company, Ebco Technologies, is also proposing this type of cyclotron. Two units of their TR30 are installed around the world.

The first CYCLONE 30's used a $2 \mathrm{~mA}$ external multicusp ion source made by IBA, and a $25 \mathrm{~kW}$ output R.F. final amplifier. The extracted beam intensity $350 \mu \mathrm{A}$ guaranteed, with actual maximum currents varying from machine to machine between $450 \mu \mathrm{A}$ and $600 \mu \mathrm{A}$. There is also some interest for higher intensities, so today several high intensity versions of CYCLONE 30 are also proposed, based on more powerful RF amplifiers, four high pumping speed cryopumps, and higher intensity ion sources $\left(7 \mathrm{~mA}\right.$ or even $\left.25 \mathrm{~mA}^{-} \mathrm{H}^{-}\right)$.

\section{CYCLOTRONS SPECIFICALLY DESIGNED FOR POSITRON EMISSION TOMOGRAPHY CENTRES}

Positron Emission Tomography (PET) is a powerful diagnostic tool at the forefront of modern medical imaging. PET images biological function and physiology instead of anatomy. It uses short-lived radioactive isotopes $\left({ }^{11} \mathrm{C},{ }^{13} \mathrm{~N},{ }^{15} \mathrm{O},{ }^{18} \mathrm{~F}\right)$ which can be produced by lowenergy cyclotrons $(<20 \mathrm{MeV})$ accelerating protons and/or deuterons.

A cyclotron of the type of those presented in the above paragraph may also be used to produce the radioisotopes needed for PET, but the very short life-time (a few minutes half-life) of most radioisotopes used in PET makes it convenient and appropriate to place the isotope production equipment very close to the scanners. Moreover, lower energies are used compared to the 20 $30 \mathrm{MeV}$ typically needed to produce radioisotopes for nuclear medicine. A range of turn-key systems have therefore been developed by a number of companies, including targets and automated chemistry modules in addition to cyclotrons, and specifically covering each segment of the PET market. These include large manufacturers of medical equipment such as General Electric or Siemens/CTI, as well as accelerator-dedicated companies such as IBA [3] or Ebco.

Typical energies for this application are in the range of 10 to $18 \mathrm{MeV}$. Some of the cyclotrons accelerate only protons, while others produce both protons and deutons. Most of these accelerators can be self-shielded and accelerate negative ions. The extraction is done by the stripping method. Several targets can be used, located either internally or externally. Typical maximum extracted currents vary between 50 and $100 \mu \mathrm{A}$. The systems are fully automated, from cyclotron operation to radiochemistry, with a particular emphasis on targetry and chemistry.

\section{HIGH INTENSITY CYCLOTRONS FOR THE PRODUCTION OF RADIOISOTOPES FOR BRACHYTHERAPY}

In addition to the radioisotopes used for imaging and diagnosis in nuclear medicine and PET, some radioisotopes are used for cancer therapy. The beam currents needed for the production of these isotopes are much higher and are generally expressed in milliampere. In 1992 IBA was asked to develop a very high intensity, $18 \mathrm{MeV}$ cyclotron for the production of the radioisotope ${ }^{103} \mathrm{Pd}$. This radioisotope is marketed, in small sealed sources, for example for the local treatment of prostate cancer, by the company Theragenics in Atlanta $(\mathrm{Ga})$. It can be produced by a $(\mathrm{p}, \mathrm{n})$ reaction on Rhodium, a good material for internal target. Because of the low reaction yield, the production of the ${ }^{103} \mathrm{Pd}$ isotope for cancer treatment requires a proton current in excess of one milliampere, at an energy of 15 to $18 \mathrm{MeV}$. An internal target being acceptable for this application, IBA designed a very high current positive ion, internal target cyclotron: the CYCLONE 18+. This cyclotron has demonstrated the possibility to provide continuous, unattended operation at 
$2 \mathrm{~mA}$ on target for one week irradiation's. An interesting feature of this cyclotron is the beam loading factor, reaching $80 \%$ of the total R.F. power, with only $20 \%$ being used to maintain the accelerating field. A $7 \mathrm{~mA}$ current was observed during factory tests on a beam dump at lower energy (the maximum current was actually limited by the melting of the beam dump, not by the ion source or space charge limits) [4].

\section{ACCELERATORS FOR CONVENTIONAL RADIOTHERAPY}

Externally produced photon beams, or X-rays, contribute to more than $80 \%$ of all radiotherapy treatments. In addition, electron-beam therapy is used in 10 to 15 percent of cases. These beams are currently produced mainly by $\mathrm{rf}$ linear accelerators, which have today a dominant place on the world market of medical accelerators for external beam radiotherapy. According to [5], the total number of radiotherapy electron accelerators was around 4000 in 1996 . This success can be explained by the effectiveness of the treatment and by the fact that conventional radiotherapy is the less expensive method of cancer treatment compared to other modalities such as surgery or chemotherapy for example.

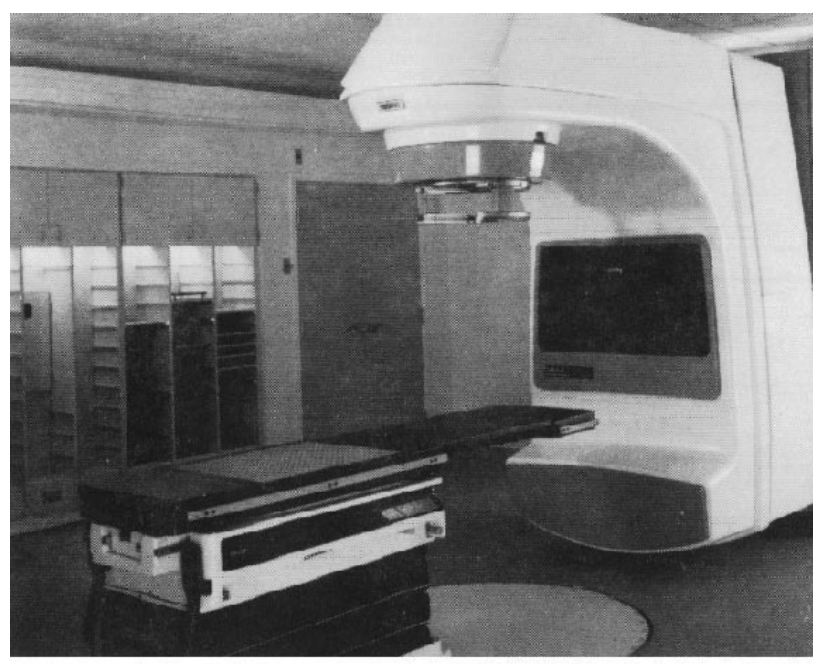

Fig. 2. RF linear accelerator manufactured by Varian [5]

The design of the first generation of Linacs in the mid1950 was based on the in-line position of the accelerating waveguide in the movable treatment head [5]. In the sixties, the available machines had an horizontally mounted accelerating waveguide, with the advantage of generating higher energy beams but needing a $90^{\circ}$ bending magnet. Later on, in the seventies, waveguides with a travelling wave were replaced by structures using a standing wave. This made it possible to increase the linear gradients and therefore to obtain smaller accelerators for the same energy. In the first models the accelerating structure was still horizontal and used a $270^{\circ}$ achromatic magnet, but nowadays, some models use inline waveguides, thus avoiding the need for bending magnets. The full rotating head of these machines include several devices such as X-rays conversion targets, collimators, dosimetry devices, etc. Multi-energy accelerators are now also available, offering the possibility of producing two photon energies as well as several electron energies. Typical values are 6 and 10, 15 or $18 \mathrm{MV}$ for X-rays, and six electron energies between 4 and 20 or $22 \mathrm{MeV}$. Well known manufacturers include companies such as Varian, Siemens, Philips and General Electric, among others.

\section{ACCELERATORS FOR PROTON AND NEUTRON THERAPY}

\section{A Proton Therapy}

The advantage of protons in radiation therapy, especially their ballistic accuracy, is widely acknowledged. However, the development of proton therapy as a clinical tool has been hampered by the complexity, the size and the cost of proton therapy facilities. Indeed, the medical use of protons require high energy accelerators not adapted, up to now, to the hospital environment. Pioneering institutions had to work with complex, inadequate equipment designed for research in nuclear physics. Today things are changing and a number of proton therapy systems specifically designed for inhospital operation are under installation or already in operation. One of them is the synchrotron-based proton therapy system at Loma Linda, California. It was jointly built by a team of the Fermi National Accelerator Laboratory in Chicago, Illinois, the Loma Linda University Medical Center and SAIC (Science Applications International Corporation), a private company based in San Diego. The Loma Linda system includes an accelerator as well as a beam transport system and a number of gantries allowing to irradiate the tumour from any direction. The accelerator system includes an RFQ injector (2 MeV) and a synchrotron delivering a 70 to $250 \mathrm{MeV}$ pulsed beam.

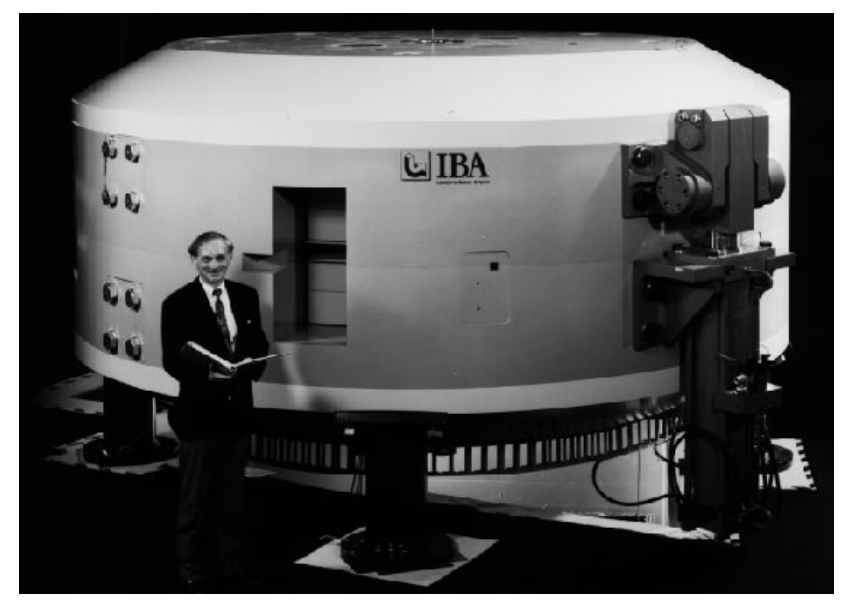

Fig. 3. A $235 \mathrm{MeV}$ cyclotron for proton therapy

However, the first proton therapy system specifically designed for in-hospital operation and to be installed as a commercial system is a cyclotron-based system. IBA has indeed integrated the technologies developed for its low and medium energy cyclotrons into a high energy system dedicated to proton therapy. The objective was to meet all the clinical specifications of a state-of-the-art proton 
therapy facility in the most simple, reliable and cost effective manner. The main elements of the IBA integrated system are the following [5]: a compact 235 $\mathrm{MeV}$ isochronous cyclotron, a short energy selection system transforming the fixed energy beam extracted from the cyclotron into a variable $(70-235 \mathrm{MeV})$ energy beam, one or more isocentric gantries fitted with a nozzle, a system consisting of one or more horizontal beam lines, a global control system including an accelerator control unit and three independent but networked therapy control stations, a global safety management system independent of the global control system, and a robotic patient positioning system. Two of these systems are presently under installation: one at the Massachusetts General Hospital of the Harvard Medical School in Boston, MA (see [6] as well as another paper presented at this conference), and another one at the National Cancer Center near Tokyo, in Japan.

\section{$B$ Neutron Therapy}

Neutron therapy is more effective than conventional radiation for certain advanced tumours because it has the ability to kill tumours cells which are low in oxygen and often become resistant to the usual forms of radiation. A prototype of a Neutron Therapy System developed by Dr. Blosser at the Michigan State University is in operation since several years at the Harper Hospital in Detroit, MI. The system, which is also commercialised by IBA, consists of an ultra-compact, superconducting cyclotron which rotates around the patient. The cyclotron is indeed mounted on a rotating gantry allowing 360 degrees rotation around the patient. It accelerates deuterons up to $50 \mathrm{MeV}$ to produce neutrons on an internal Beryllium target.

\section{INDUSTRIAL APPLICATIONS WITH ELECTRON BEAMS}

Electron beams are used in industry for several applications including the sterilization of medical disposable, the improvement of properties of polymer materials, and food treatment. The energy of the beam, together with the product density, determines the thickness of the products which can be treated. At a fixed energy, the treatment capacity will depend on the power of the beam. The energy of an electron accelerator is therefore a key parameter for the classification of the several types of accelerators used in this field.

At very low energy (few hundreds $\mathrm{keV}$ ), typical accelerators are single gap machines. These include machines with point cathodes and scanners which scan the beam over the surface of the product to be treated, and linear cathode machines which emit electrons from a linear cathode irradiating the product over its entire surface. Another development is the WIP (Wire Induced Plasma) system in which a stream of ions generate in a plasma secondary electrons which are consequently accelerated. The advantage of these single gap accelerators is the fact that they are self-shielded, have a small size, and produce high beam currents. They are well adapted for many surface and thin layer applications.
At low energy (say up to $3 \mathrm{MeV}$ ), multi-gap accelerators with electron beam tubes and scanners are used. Different high voltage generating principles can be used. Typical accelerators in this energy range include ICT (Insulated Core Transformer), dynamitrons and Cockroft-Walton generators. Well known commercial companies in this field include High Voltage Engineering, Radiation Dynamics and Nissin-High Voltage, among others. The machines are larger than those used in the very low energy field, and they need concrete radiation shielding. Some of them produce high power beams $(100 \mathrm{~kW})$. A typical application in this low energy range is the crosslinking of several types of industrial components (cables, pipes, ...). Accelerators in this energy range are the classical equipement in the electron beam market, with more than 100 machines in operation.

At medium energy (between 3 and $5 \mathrm{MeV}$ ), most of the available accelerators are of the dynamitron or the Cockroft-Walton types. Output power levels are in the range of ten to hundred $\mathrm{kW}$. These machines require meter thick concrete shielding and are typically used for crosslinking and also for sterilization of packaged medical disposable products.

The high energy category includes energies between 5 and $10 \mathrm{MeV}$. Up to a few years, typical machines in this energy range were Linacs such as those proposed by AECL, Canada. These Linacs are RF devices producing pulsed beams. Today, installed systems are limited to moderate powers $(10$ to $50 \mathrm{~kW})$. Since three years, however, IBA entered the field of high energy, high current electron accelerators for sterilization and other industrial applications with its RHODOTRON [7]. This new accelerator concept, developed at the CEA in Saclay (France), was licensed to IBA. RHODOTRON accelerators are based on an innovative concept of using a single "recirculating cavity" to provide high energy (up to $10 \mathrm{MeV}$ ) / high power (up to $200 \mathrm{~kW}$ ) electron beams, with high electrical efficiency. Three Rhodotron models are now available, ranging from 25 to $150 \mathrm{~kW}$ guaranteed beam power at $10 \mathrm{MeV}$. The Rhodotron technology opens the doors to industrial applications for which high energy / high current electron beams are needed: crosslinking of plastics, polymerization of composite materials, sterilization of medical products, food treatment, etc. 


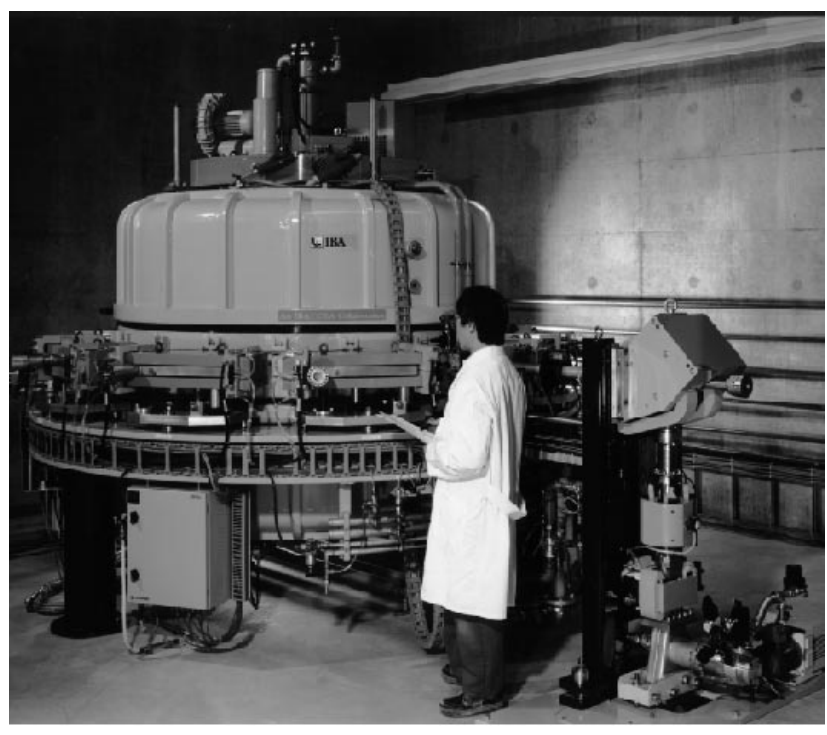

Fig. 4. A RHODOTRON accelerator from IBA

\section{REFERENCES}

[1] S. M. Qaim, "Nuclear data relevant to cyclotron produced shortlived medical radioisotopes", Radiochimica Acta 30, pp. 147-162, 1982

[2] Y. Jongen et al., "High Intensity Cyclotrons for Radioisotope Production", Proceedings of the $14^{\text {th }}$ International Conference on Cyclotrons and their Applications, Cape Town, South Africa, 1995, pp. $115-119$

[3] M. Abs et al., "The IBA PET-dedicated Cyclotrons: Main Features and Improvements", Proceedings of the 14th International Conference on Cyclotrons and their Applications, Cape Town, South Africa, 1995, pp. 120-123

[4] Y. Jongen, "Extremely High Intensity Cyclotrons for Radioisotope Production", proceedings of the EPAC'94 Conference, London, 1994

[5] W. Scharf et al., "Medical Accelerators in Radiotherapy: Past, Present and Future", Physica Medica - Vol.XII, N.4, 1996

[6] Y. Jongen et al., "The proton therapy system for the NPTC", Proceedings of the 4th European Conference on Accelerators in Applied Research and Technology, Zürich, August 1995

[7] Y. Jongen et al., "Rhodotron accelerators for industrial electronbeam processing: a progress report", Proceedings of the EPAC'96 Conference, Sitges, Barcelona, 1996 